\title{
Numerical Solution of a System SEIR Nonlinear ODEs by Runge-Kutta Fourth Order Method
}

\author{
A.A.M. Hassan \\ Department of Mathematics, \\ Faculty of Science \\ Zagazig University
}

\author{
S.A. Hoda Ibrahim \\ Department of Mathematics, \\ Faculty of Science \\ Zagazig University
}

\author{
Mohamed. G.M. Ibrahim \\ Demonstrator at Thebes academy \\ Faculty of Science \\ Zagazig University
}

\begin{abstract}
In this paper, we introduce the numerical solution of the system of SEIR nonlinear ordinary differential equations, which are studied the effect of vaccine on the HIV (Human Immunology virus). We obtained the numerical solutions on stable manifolds by Runge-Kutta fourth order method.
\end{abstract}

\section{Keywords}

SEIR model; nonlinear ordinary differential equations; RungeKutta fourth order method.

\section{INTRODUCTION}

The SEIR (Susceptible-Exposed-Infected- Removed) ordinary differential equations, developed by Ronald Ross, William Hamer, and others in the early twentieth century [2], Goufo, etal.[3] have presented a fractional SEIR meta population system modeling to the dynamics between four distinct cities (patches) by using Jacobin matrix.

There are many authors solved nonlinear ordinary differential equations by using Runge-Kutta method, see [4, 5].

The objective of this paper is to solving the fractional SEIR Meta population system by using Runge-Kutta fourth order method. The rest of this paper arranged as the following, in Sec. 2, the description of the Runge-Kutta fourth order method. In Sec. 3, application of Runge-Kutta fourth order method for SEIR model. In Sec.4, conclusion

2. DESCRIBTION OF THE RUNGEKUTTA FOURTH ORDER METHOD [4].

Suppose we have a system of ordinary differential equations as the form.

$$
\begin{aligned}
& y_{1}^{\prime}=f_{1}\left(x, y_{1}, y_{2}, \ldots, y_{n}\right), \\
& y_{2}^{\prime}=f_{2}\left(x, y_{1}, y_{2}, \ldots, y_{n}\right), \\
& \vdots \\
& y_{n}^{\prime}=f_{n}\left(x, y_{1}, y_{2}, \ldots, y_{n}\right),
\end{aligned}
$$

With the initial conditions

$$
y_{i}(0)=y_{0}^{i}, i=0,1, \ldots, n
$$

Putting them as the vector form:

$Y_{n}^{\prime}=F_{n}(x, y), Y\left(x_{0}\right)=y_{0}$,

where

$$
Y(x)=\left[\begin{array}{l}
y_{1} \\
y_{2} \\
\vdots \\
y_{n}
\end{array}\right], F(x, y)=\left[\begin{array}{l}
f_{1} \\
f_{2} \\
\vdots \\
f_{n}
\end{array}\right] .
$$

And the Runge-Kutta fourth order as:

$$
\begin{aligned}
& Y_{1(m+1)}=Y_{1(m)}+k_{10}, \\
& Y_{2(m+1)}=Y_{2(m)}+k_{20}, \\
& \vdots \\
& Y_{n(m+1)}=Y_{n(m)}+k_{n 0},
\end{aligned}
$$

where

$$
\begin{aligned}
& k_{10}=\frac{h}{6}\left(k_{11}+2\left(k_{12}+k_{13}\right)+k_{14}\right), \\
& k_{20}=\frac{h}{6}\left(k_{21}+2\left(k_{22}+k_{23}\right)+k_{24}\right), \\
& \vdots \\
& k_{n 0}=\frac{h}{6}\left(k_{n 1}+2\left(k_{n 2}+k_{n 3}\right)+k_{n 4}\right) .
\end{aligned}
$$

And

$$
\begin{aligned}
& k_{11}=f\left(t_{0}, Y_{10}, Y_{20}, \ldots, Y_{n 0}\right), \\
& k_{12}=f\left(t_{0}+\frac{h}{2}, \mathrm{Y}_{10}+\frac{k_{11}}{2}, \ldots, \mathrm{Y}_{n 0}+\frac{k_{11}}{2}\right), \\
& k_{13}=f\left(t_{0}+\frac{h}{2}, \mathrm{Y}_{10}+\frac{k_{12}}{2}, \ldots, \mathrm{Y}_{n 0}+\frac{k_{12}}{2}\right), \\
& k_{14}=f\left(t_{0}+\frac{h}{2}, \mathrm{Y}_{10}+k_{13}, \ldots, \mathrm{Y}_{n 0}+k_{13}\right) .
\end{aligned}
$$


And

$$
\begin{aligned}
& k_{21}=f\left(t_{0}, Y_{10}, Y_{20}, \ldots, Y_{n 0}\right), \\
& k_{22}=f\left(t_{0}+\frac{h}{2}, \mathrm{Y}_{10}+\frac{k_{21}}{2}, \ldots, \mathrm{Y}_{n 0}+\frac{k_{21}}{2}\right), \\
& k_{23}=f\left(t_{0}+\frac{h}{2}, \mathrm{Y}_{10}+\frac{k_{22}}{2}, \ldots, \mathrm{Y}_{n 0}+\frac{k_{22}}{2}\right), \\
& k_{24}=f\left(t_{0}+\frac{h}{2}, \mathrm{Y}_{10}+k_{23}, \ldots, \mathrm{Y}_{n 0}+k_{23}\right) . \\
& \vdots \\
& k_{n 1}=f\left(t_{0}, Y_{10}, Y_{20}, \ldots, Y_{n 0}\right), \\
& k_{n 2}=f\left(t_{0}+\frac{h}{2}, \mathrm{Y}_{10}+\frac{k_{n 1}}{2}, \ldots, \mathrm{Y}_{n 0}+\frac{k_{n 1}}{2}\right), \\
& k_{n 3}=f\left(t_{0}+\frac{h}{2}, \mathrm{Y}_{10}+\frac{k_{n 2}}{2}, \ldots, \mathrm{Y}_{n 0}+\frac{k_{n 2}}{2}\right), \\
& k_{n 4}=f\left(t_{0}+\frac{h}{2}, \mathrm{Y}_{10}+k_{n 3}, \ldots, \mathrm{Y}_{n 0}+k_{n 3}\right) .
\end{aligned}
$$

\section{APPLICATION OF RUNGE-KUTTA FOURTH ORDER METHOD FOR SEIR MODEL}

In this section, we will apply the numerical solution for a system of differential equations [4,5], that mentioned of this method can be obtained from [4,5], SEIR model [3]. We formulate here the system modeling the fractional temporal spread of measles in a human population. In this model, a population supposed constant is divided into different classes, disjoint and based on their disease status. At time $t, S=S(t)$ is the fraction of population representing individual susceptible to measles, $\mathrm{E}=\mathrm{E}(\mathrm{t})$ is the fraction of population representing individuals exposed to measles, $\mathrm{I}=\mathrm{I}(\mathrm{t})$ is the fraction of population representing individuals infectious with measles, and $R=R(t)$ is the fraction of population representing individuals that recovered from measles. We assume that all recruitment is done by birth into the class of susceptible and occurs at constant birth rate $b$. The rate constant for no disease related death is $\mu$; thus $1 / \mu$ is the average lifetime. We use the standard mas balance incidence expressions $\beta S I$ to indicate successful transmission of measles due to effective contacts dynamics in the population by infectious individuals. Once infected, a fraction of exposed people becomes infectious with a constant rate $\sigma$, so that $1 / \sigma$ is the average incubation period. Some infectious individuals will recover after a treatment or a certain period of time at a rate constant $\xi$, making $1 / \xi$ the average infectious period. $\frac{d S}{d t}=b-(\beta I+\mu) S$,

$\frac{d E}{d t}=\beta S I-(\sigma+\mu) \mathrm{E}$,

$\frac{d I}{d t}=\sigma \mathrm{E}-(\xi+\mu) \mathrm{I}$

$\frac{d R}{d t}=\xi I-\mu R$.

With the initial conditions

$S(0)=1, E(0)=0, I(0)=1$ And $R(0)=0$.

And the numerical solution of SEIR (ODEs) can be determined by Runge-Kutta fourth order method.

Put

$b=0, \beta=0.5, \sigma=0.75, \mu=0.25, \xi=0.75$.

Equation (3.1) can take the form

$$
\begin{aligned}
& \frac{d S}{d t}=-(0.5 I+0.25) S, \\
& \frac{d E}{d t}=0.5 S I-\mathrm{E}, \\
& \frac{d I}{d t}=0.75 \mathrm{E}-\mathrm{I}, \\
& \frac{d R}{d t}=0.75 I-0.25 R .
\end{aligned}
$$

The solution can be obtained by the following relations

$S=S_{0}+k_{10}, E=E_{0}+k_{20}, I=I_{0}+k_{30}$ and

$R=R_{0}+k_{30}$

where

$$
\begin{aligned}
& k_{10}=\frac{h}{6}\left(k_{11}+2\left(k_{12}+k_{13}\right)+k_{14}\right), \\
& k_{20}=\frac{h}{6}\left(k_{21}+2\left(k_{22}+k_{23}\right)+k_{24}\right), \\
& k_{30}=\frac{h}{6}\left(k_{31}+2\left(k_{32}+k_{33}\right)+k_{34}\right), \\
& k_{40}=\frac{h}{6}\left(k_{41}+2\left(k_{42}+k_{43}\right)+k_{44}\right) .
\end{aligned}
$$


and

$$
\begin{aligned}
& k_{11}=f\left(t_{0}, S_{0}, E_{0}, I_{0}, R_{0}\right), \\
& k_{12}=f\left(t_{0}+\frac{h}{2}, S_{0}+\frac{k_{11}}{2}, \mathrm{E}_{0}+\frac{k_{11}}{2}, R_{0}+\frac{k_{11}}{2}\right), \\
& k_{13}=f\left(t_{0}+\frac{h}{2}, S_{0}+\frac{k_{12}}{2}, \mathrm{E}_{0}+\frac{k_{12}}{2}, R_{0}+\frac{k_{12}}{2}\right), \\
& k_{14}=f\left(t_{0}+\frac{h}{2}, S_{0}+k_{13}, \mathrm{E}_{0}+k_{13}, R_{0}+k_{13}\right), \\
& k_{21}=f\left(t_{0}, S_{0}, E_{0}, I_{0}, R_{0}\right), \\
& k_{22}=f\left(t_{0}+\frac{h}{2}, S_{0}+\frac{k_{21}}{2}, \mathrm{E}_{0}+\frac{k_{21}}{2}, R_{0}+\frac{k_{21}}{2}\right), \\
& k_{23}=f\left(t_{0}+\frac{h}{2}, S_{0}+\frac{k_{22}}{2}, \mathrm{E}_{0}+\frac{k_{22}}{2}, R_{0}+\frac{k_{22}}{2}\right), \\
& k_{24}=f\left(t_{0}+\frac{h}{2}, S_{0}+k_{23}, \mathrm{E}_{0}+k_{23}, R_{0}+k_{23}\right),
\end{aligned}
$$$$
k_{31}=f\left(t_{0}, S_{0}, E_{0}, I_{0}, R_{0}\right),
$$$$
k_{32}=f\left(t_{0}+\frac{h}{2}, S_{0}+\frac{k_{31}}{2}, \mathrm{E}_{0}+\frac{k_{31}}{2}, R_{0}+\frac{k_{31}}{2}\right),
$$$$
k_{33}=f\left(t_{0}+\frac{h}{2}, S_{0}+\frac{k_{32}}{2}, \mathrm{E}_{0}+\frac{k_{32}}{2}, R_{0}+\frac{k_{32}}{2}\right) \text {, }
$$$$
k_{34}=f\left(t_{0}+\frac{h}{2}, S_{0}+k_{33}, \mathrm{E}_{0}+k_{33}, R_{0}+k_{33}\right) \text {, }
$$

and

$$
\begin{aligned}
& k_{41}=f\left(t_{0}, S_{0}, E_{0}, I_{0}, R_{0}\right), \\
& k_{42}=f\left(t_{0}+\frac{h}{2}, S_{0}+\frac{k_{41}}{2}, \mathrm{E}_{0}+\frac{k_{41}}{2}, R_{0}+\frac{k_{41}}{2}\right), \\
& k_{43}=f\left(t_{0}+\frac{h}{2}, S_{0}+\frac{k_{42}}{2}, \mathrm{E}_{0}+\frac{k_{42}}{2}, R_{0}+\frac{k_{42}}{2}\right), \\
& k_{44}=f\left(t_{0}+\frac{h}{2}, S_{0}+k_{43}, \mathrm{E}_{0}+k_{43}, R_{0}+k_{43}\right) .
\end{aligned}
$$

\section{Step 1:}

We start with the initial conditions $\mathrm{t}=0.25$, $S_{0}=1, E_{0}=0, I_{0},=1$ and $R_{0}=0$.

By substituting in equations (3.3) we can get

$k_{11}=f(0.25,1,0,1,0)=\left[\begin{array}{l}-0.75 \\ 0.5 \\ -1 \\ 0.75\end{array}\right]$,

$$
=\left[\begin{array}{l}
-0.3125 \\
-0.09375 \\
-0.3125 \\
0.28125
\end{array}\right]
$$$$
k_{12}=f(0.25,0.625,0.25,0.5,0.375)
$$

$$
k_{13}=f(0.25,0.843,-0.046,0.843,0.140)
$$

$$
=\left[\begin{array}{l}
-0.56689 \\
0.40283 \\
-0.87891 \\
0.59766
\end{array}\right]
$$

$$
k_{14}=f(0.25,00.433,0.402,0.121,0.597)
$$

$$
=\left[\begin{array}{l}
-0.1345 \\
-0.37661 \\
0.18103 \\
-0.058598
\end{array}\right] \text {, }
$$

Then

$$
\begin{aligned}
k_{10}= & \frac{0.25}{6}(-0.75-2(0.3125-0.566)-0.314) \\
& =-0.1101366667, \\
S_{1}= & 1-0.1101366667=0.8898633333, \\
k_{20}= & \frac{0.25}{6}(0.5+2(-0.09375+0.40283)-0.37661) \\
= & 0.03089791667, \\
E_{1}= & 0+0.03089791667=0.03089791667,
\end{aligned}
$$

$$
\begin{aligned}
k_{30} & =\frac{0.25}{6}(-1-2(0.3125+0.87891)+0.18103) \\
& =-0.1334079167,
\end{aligned}
$$

$I_{1}=1-0.1334079167=0.8665920833$, and

$$
\begin{aligned}
k_{40} & =\frac{0.25}{6}(0.75+2(0.28125+0.59766)-0.058598) \\
& =0.1020509167, \\
R_{1} & =0+0.1020509167=0.1020509167 .
\end{aligned}
$$

Step 2: 
At $\quad \mathrm{t}=0.5, \quad \mathrm{~h}=0.25, \quad S_{1}=0.8898633333$,

$E_{1}=0.03089791667, I_{1},=0.8665920833$ and $R_{1}=0.1020509167$.

By substituting in equations (3.3) we can get

$$
\begin{aligned}
k_{11}= & f\left(0.5,0.889,3.089 \times 10^{-2}, 0.866,0.102\right) \\
= & {\left[\begin{array}{l}
-0.60804 \\
0.35467 \\
-0.84342 \\
0.62443
\end{array}\right], }
\end{aligned}
$$$$
k_{12}=f(0.5,0.75148,0.208,0.444,0.414)
$$$$
=\left[\begin{array}{l}
-0.27677 \\
-7.7916 \times 10^{-2} \\
-0.28871 \\
0.30815
\end{array}\right],
$$$$
k_{13}=f\left(0.5,0.843,-8.06 \times 10^{-3}, 0.722,0.256\right)
$$$$
=\left[\begin{array}{l}
-0.51563 \\
0.31276 \\
-0.72829 \\
0.47765
\end{array}\right],
$$

Also,

$k_{14}=f(0.5,0.37423,0.34366,0.1383,0.5797)$

$$
=\left[\begin{array}{l}
-0.11944 \\
-0.31778 \\
0.11945 \\
-0.0412
\end{array}\right]
$$

we have

$$
\begin{array}{rl}
k_{10} & =\frac{0.25}{6}(-0.608-2(0.276+0.515)-0.11) \\
& =-9.6345 \times 10^{-2}, \\
S_{2}= & 0.8898633333-9.6345 \times 10^{-2}=0.79351833, \\
k_{20}= & \frac{0.25}{6}(0.354+2(0.312-0.077)-0.317) \\
& =2.110741667 \times 10^{-2}, \\
\mathrm{E}_{2} & 0.03089791667+2.110741667 \times 10^{-2} \\
& =5.200538337 \times 10^{-2}, \\
\mathrm{k}_{30} & =\frac{0.25}{6}(-0.843-2(0.288+0.728)+0.119)
\end{array}
$$

$$
\begin{aligned}
& =-0.1149154167, \\
I_{2}= & 0.8665920833-0.1149154167 \\
& =0.7516766666,
\end{aligned}
$$

\section{Step 3:}

At $\quad \mathrm{t}=0.75, \quad \mathrm{~h}=0.25, \quad S_{2}=0.7935183333$, $E_{2}=5.200538337 \times 10^{-2}, I_{2}=0.7516766666$, and $R_{2}=0.1918355$.

Substituting in equations (3.3) we can get the following:

$$
\begin{aligned}
k_{11}= & f\left(0.75,0.793,5.2 \times 10^{-2}, 0.751,0.191\right) \\
& =\left[\begin{array}{l}
-0.49661 \\
0.4623 \\
-0.71267 \\
0.51580
\end{array}\right],
\end{aligned}
$$

$$
\begin{aligned}
k_{12}= & f(0.75,0.545,0.175,0.395,0.449) \\
& =\left[\begin{array}{l}
-0.24407 \\
-6.7348 \times 10^{-2} \\
-0.264 \\
0.18407
\end{array}\right]
\end{aligned}
$$

$$
\begin{aligned}
k_{13}= & f\left(0.75,0.671,1.8 \times 10^{-2}, 0.619,0.283\right) \\
& =\left[\begin{array}{l}
-0.37592 \\
0.18972 \\
-0.60593 \\
0.39379
\end{array}\right],
\end{aligned}
$$

$$
k_{14}=f(0.75,0.417,0.241,0.145,0.585)
$$

$$
=\left[\begin{array}{l}
-0.13483 \\
-0.21130 \\
3.5548 \times 10^{-2} \\
-3.7095 \times 10^{-2}
\end{array}\right],
$$


Also, we have

$$
\begin{aligned}
k_{10}= & \frac{0.25}{6}(-0.496-2(0.244+0.375)-0.314) \\
& =-7.797583333 \times 10^{-2}, \\
S_{3}= & 0.793518333-7.797583333 \times 10^{-2} \\
= & 0.7155425, \\
k_{20}= & \frac{0.25}{6}(0.24623+2(-0.0673+0.189)-0.211) \\
= & 1.165308333 \times 10^{-2}, \\
E_{3}= & 5.200538337 \times 10^{-2}+1.165308333 \times 10^{-2} \\
= & 6.36584667 \times 10^{-2}, \\
k_{30}= & \frac{0.25}{6}(-0.712-2(0.264+0.605)+0.0355) \\
& =-0.1007075833, \\
I_{3}= & 0.7516766666-0.1007075833 \\
= & 0.65096908333, \\
\text { and } & \\
k_{40}= & \frac{0.25}{6}(0.515+2(0.184+0.393)-0.037) \\
= & 6.810104167 \times 10^{-2}, \\
R_{3}= & 0.1918355+6.810104167 \times 10^{-2} \\
= & 0.2599365417 \\
S_{1} &
\end{aligned}
$$

Step 4:

At $\quad \mathrm{t}=1, \quad \mathrm{~h}=0.25, \quad S_{3}=0.7155425$,

$E_{3}=0.0636584667, I_{3}=0.6509690833$,

and $R_{3}=0.2599365417$.
Substituting in equations (3.3) we can get the following:

$$
\begin{aligned}
k_{11}= & f(1,0.7155,0.0636,0.6509,0.2599) \\
= & {\left[\begin{array}{l}
-0.41178 \\
0.16924 \\
-0.60323 \\
0.42324
\end{array}\right], }
\end{aligned}
$$$$
k_{12}=f(1,0.5096,0.1482,0.3493,0.4715)
$$$$
=\left[\begin{array}{l}
-0.21644 \\
-5.9257 \times 10^{-2} \\
-0.23814 \\
0.14412
\end{array}\right],
$$

$$
k_{13}=f(1,0.6073,0.034,0.531,0.332)
$$

$$
=\left[\begin{array}{l}
-0.31335 \\
0.12749 \\
-0.50638 \\
0.31593
\end{array}\right],
$$

$$
\begin{aligned}
k_{41} & =f(1,0.4021,0.1911,0.1445,0.5758) \\
& =\left[\begin{array}{l}
-0.12962 \\
-0.16207 \\
-1.2275 \times 10^{-2} \\
-3.5525 \times 10^{-2}
\end{array}\right] .
\end{aligned}
$$

Also, we have

Obvious table 3.1.1:

\begin{tabular}{|c|c|c|c|c|}
\hline$t_{i}$ & $\begin{array}{c}\text { Numerical Solution } \\
\text { of } \mathbf{S}_{n} \text { groups }\end{array}$ & $\begin{array}{c}\text { NumericalSolution } \\
\text { of } \mathbf{E}_{n} \text { groups }\end{array}$ & $\begin{array}{c}\text { NumericalSolution } \\
\text { of } \mathbf{I}_{n} \text { groups }\end{array}$ & $\begin{array}{c}\text { NumericalSolution } \\
\text { of } \mathbf{R}_{n} \text { groups }\end{array}$ \\
\hline 0 & 1 & 0 & 1 & 0 \\
\hline 0.25 & 0.8898633333 & 0.03089791667 & 0.8665920833 & 0.1020509167 \\
\hline 0.5 & 0.7935183333 & 0.05200538337 & 0.7516766666 & 0.1918355 \\
\hline 0.75 & 0.7155425 & 0.0636584667 & 0.6509690833 & 0.2599365417 \\
\hline 1 & 0.648835 & 0.06964330003 & 0.5637400208 & 0.3144288334 \\
\hline
\end{tabular}




$$
\begin{aligned}
k_{10} & =\frac{0.25}{6}(-0.411-2(0.216+0.313)-0.129) \\
& =-6.67 .75 \times 10^{-2}, \\
S_{4}= & 0.7155425-6.67075 \times 10^{-2} \\
& =0.648835, \\
k_{20} & =\frac{0.25}{6}(0.169+2(-0.059+0.127)-0.162) \\
& =5.5984833333 \times 10^{-3}, \\
E_{4} & =6.6584667 \times 10^{-2}+598483333 \times 10^{-3} \\
& =0.06964330003, \\
k_{30} & =\frac{0.25}{6}(-0.632-2(0.2381+0.5063)-0.0012) \\
& =-8.72290625 \times 10^{-2}, \\
I_{4}= & 0.650960833-8.72290625 \times 10^{-2} \\
& =0.5637400208, \\
\text { and } & \\
k_{40} & =\frac{0.25}{6}(0.4232+2(0.1441+0.3159)-0.0355) \\
& =5.449229167 \times 10^{-2}, \\
R_{4}= & 0.2599365417+5.449229167 \times 10^{-2} \\
= & 0.3144288334 .
\end{aligned}
$$

\section{CONCLUSION}

In this article, we presented the Runge-Kutta fourth order method for SEIR nonlinear ODEs. We tested the method for $\delta t=0,0.25,0.5,0.75$ and 1 , we find that the results are more accurate and efficiency.

\section{REFERENCES}

[1] L. Bubniakov, the Mathematics of Infectious Diseases, Bratislav 2007.

[2] H. Weiss, the SEIR model and the foundations of public health, Materials Mathematics, vol 3(17), pp. (18871097), 2013

[3] E. F. D. Goufo, S. C. O. Noutchie, and S. Mugisha, a Fractional SEIR Epidemic Model for Spatial and Temporal Spread of Measles in Meta populations, Abstract and Applied Analysis, Vol 7, 2014.

[4] B. S. Desale and N. R. Dasre, Numerical Solution of the System of Six Coupled Nonlinear ODEs by Runge-Kutta Fourth Order Method, Applied Mathematical Sciences, Vol. 7, p(287 - 305),2013.

[5] Z. Kalogiratou, T. Monovasilis and T. E. Simos, Simplistic Runge-Kutta-Nystrom Methods with PhaseLag Oder 8 and Infinity, Applied Mathematics \& Information Sciences, vol. 3,p(1105-1112), 2015. 